\title{
Bushfire conditions under a warming climate - the value of regional climate modelling
}

\author{
$\underline{\text { M.R. Grose }}^{\text {a }}$, P. Fox-Hughes ${ }^{\text {b }}$ and N.L. Bindoff ${ }^{\text {ac d }}$ \\ ${ }^{a}$ Antarctic Climate and Ecosystems Cooperative Research Centre (ACE CRC), Hobart Tasmania \\ ${ }^{b}$ Bureau of Meteorology, Hobart Tasmania \\ ${ }^{c}$ Institute of Marine and Antarctic Studies (IMAS), Hobart Tasmania \\ ${ }^{d}$ Centre for Australian Weather and Climate Research (CAWCR), Hobart Tasmania \\ Email: michael.grose@utas.edu.au
}

Long-term changes to the climate affect the incidence of weather conditions suitable for bushfires as well as the growth and dry matter accumulation that contribute to fuel loads. Estimating the changes to fire danger with a changing climate is an essential task for the long-term planning of bushfire response. Our best tools for projecting changes to the climate system are climate models, however the coarse scale of global climate models (GCMs) does not show the regional detail of changes. In this study, we use output from the dynamical regional climate model (RCM) named conformal cubic atmospheric model (CCAM) to examine the simulation of Tasmanian fire weather, and illustrate some advantages in using RCM outputs over GCM outputs.

This study focuses on bushfire weather; the atmospheric conditions that influence the incidence of bushfire. The dynamics behind one synoptic pattern associated with elevated bushfire weather conditions are examined, and the pattern is more reliably simulated in the RCM compared to GCMs. This suggests that the greater resolution offers some advantages, and further investigation of other atmospheric dynamics associated with elevated bushfire weather conditions is justified using these model outputs. A standard index of bushfire weather, the McArthur Mark 5 Forest Fire Danger Index is calculated directly from the model output but assuming a drought factor of the maximum 10. The model output has a fine spatial scale $(0.1$ degree lat/lon) and temporal scale (3-hourly is used) and generates output variables that are directly comparable to the observations used to calculate fire weather indices. The temporal variability of FFDI at Hobart compares well to observations at all scales, from sub-daily to seasonal and inter-annual. The spatial pattern of cumulative FFDI, the frequency of events of elevated FFDI and the maximum FFDI also compare well to observations. These results suggest that the RCM simulations offer some more detail than GCMs, and are likely to be a useful tool for examining the change in fire danger indices with a warming climate.

The results indicate that the fine resolution model output has some advantages over GCM outputs. The results also suggest that after more careful validation and ground-truthing from observations, RCM output can used directly to examine simulated events of high fire danger, rather than using the projected trend in temperature, wind and relative humidity as a basis to manipulate observed datasets. These results suggest that the direct model outputs are a useful tool to examine changes to fire weather dynamics and high fire danger events with a warming climate. Further analysis of fire weather dynamics and fire danger will be pursued using these model outputs.

Keywords: Climate change, Regional climate model, Bushfire 


\section{INTRODUCTION}

Bushfire risk is dependent on numerous factors, including a large influence of climate (eg. Crimmins \& Comrie 2004). Severe bushfire weather conditions are brought by certain weather systems, and are associated with some combination of high temperature, high wind speeds and low relative humidity (RH). The prevailing climate influences the growth, structure and dryness of fuels. A change in climate will affect the frequency and intensity of periods of severe fire weather, partly through a change in the synoptic-scale features that drive them. A change in climate also influences the growth, accumulation and moisture content of the various fuels.

The effect of an anthropogenic greenhouse effect is for warmer average temperatures, and there is a general perception that this will lead to higher fire danger. However, periods of severe fire weather are outside the average, and changes to these extremes may differ from the average change, and temperature is only one component of fire danger. To get a full understanding of the effect of projected climate change on bushfire weather, it is useful to examine the dynamics of severe bushfire risk events and all associated variables. Also, the effect of climate changes on bushfire fuels must be accounted for. Climate models are the only practical tool for making climate change projections, however the typical resolution of global climate models (GCMs) is relatively coarse. Extra information regarding regional detail of projected changes can be derived using a process of downscaling using regional climate model (RCM) simulations.

This paper discusses the impact of a change in climate on the atmospheric aspects of bushfire weather at a regional scale for Tasmania. Bushfire fuels are not included in this brief analysis, but will be examined in subsequent work. Specifically, we examine the value of the greater resolution in a RCM compared to the typical GCMs for examining fire weather. We examine two aspects of fire weather that are relevant to the study site of Tasmania, Australia. A synoptic-scale pattern that leads to extreme bushfire conditions is examined in the RCM simulation, and on-the-ground fire danger simulated in the model is calculated using an example standard fire danger index.

\subsection{Dynamics of extreme fire weather events}

There are several aspects of atmospheric dynamics relevant to severe bushfire weather in Tasmania that warrant investigation. This brief paper presents only one example synoptic weather pattern as an indication of the type of dynamics that can be examined from climate model outputs. Mills (2005) identifies a specific synoptic circumstance that is associated with a significant number of the extreme fire weather events in southeastern Australia and Tasmania, including the 1983 'Ash Wednesday' fires. This synoptic pattern is a particularly strong and deep cold front that drives strong dry northwesterly winds. It can be identified by high temperatures and a strong thermal gradient at the $850 \mathrm{hPa}$ level over the Bass Strait region. Hasson et al. (2008) examined this pattern in GCM outputs, and faced several difficulties in applying the method of Mills (2005), and found a large spread between models. Some model simulations produced events with a pattern similar to that observed, whereas some models produced patterns that did not. The coarse spatial and temporal scale of GCM outputs is almost certainly one reason for the mismatch between the simulations and observations. Finer scale RCM outputs may offer advantages over GCM scale simulations in the representation of this synoptic pattern identified by Mills (2005).

\subsection{Index of fire danger}

There are numerous indices for measuring fire danger and various aspects to consider in the application of each one for any circumstance. So we can compare to other studies, and for simplicity, we examine one commonly used index, the McArthur Mark 5 forest fire danger index (FFDI, McArthur 1967).

Model outputs can be spatially and temporally coarse and have biases in their mean state or distribution of relevant variables. Therefore, there are two approaches to calculating FFDI from model output: 1) to calculate the index directly from base variables from the model outputs, with or without some 'correction factors' to align the output with observations, or 2) to calculate FFDI from a dataset of observed climate variables that have been modified according to the trend indicated by the model. This modification can be of either the mean, or a measure of mean and variability.

Williams et al. (2001) calculated FFDIs under a doubled $\mathrm{CO}_{2}$ climate for southeastern Australia using GCM outputs with 'correction factors' applied. Due to the coarse scale of GCM outputs, along with other difficulties (e.g. model output was of mean RH, not minimum RH), the model outputs required modification of both mean and variability, and the correction factors were not small (factors such as 1.3, 0.7 for Tasmanian sites). Another previous study, Hennessy et al. (2005) examined simulations using GCMs and the Conformal 
Cubic Atmospheric Model (CCAM) RCM (also used in this study) at $50 \mathrm{~km}$ resolution to calculate FFDIs. Hennessy concluded that it was not appropriate to calculate indices directly from model outputs, and used trends from the simulations to perturb historical datasets (both for mean and variability). Other recent studies of bushfire and climate scenarios have also used the approach of using perturbed climates (King et al. 2009, Bradstock et al. 2009).

\section{RCM SIMULATIONS}

Fire dynamics and FFDI were examined in a set of CCAM RCM simulations for Tasmania. Dynamical downscaling was performed in two stages: the first to a $\sim 50 \mathrm{~km}$ horizontal grid over the Australian region, the second to $\sim 10 \mathrm{~km}$ horizontal grid over the Tasmanian region. We examine a set of six simulations, each using a different GCM as input (CSIRO-Mk3.5, GFDL-CM2.0, GFDL-CM2.1, MPI/OM-ECHAM5, UKMO-HadCM3, MIROC3.2(medres)). There may be some effect of GCM selection on the simulation of fire danger for the current climate, however a larger effect is the choice of dynamically downscaling RCM. The results presented are a reflection of the CCAM RCM simulation of fire weather, not of all RCMs in general. The simulations examined here were made under the A2 emissions scenario from the special report on emissions scenario (SRES), describing high emissions and was used to show the effect of strong greenhouse forcing. However, only the baseline climate (1961-1990) is examined here, so the choice of scenario is not relevant. The downscaling methods are outlined in more detail in Corney et al. (2010).

The synoptic driver of extreme fire weather in Tasmania identified by Mills (2005) was examined in the $\sim 50$ $\mathrm{km}$ grid simulations during the baseline period 1961-1990, and FFDIs were calculated using the $\sim 10 \mathrm{~km}$ grid simulations for the period 1961-2100.

\section{CALCULATIONS AND RESULTS}

\subsection{Example synoptic driver}

Events of pre-frontal conditions that drive extreme fire weather events were identified using the approach of Mills (2005). The method uses a phase space approach based on the maximum temperature gradient at the $850 \mathrm{hPa}$ level across an analysis window over Bass Strait, and the maximum temperature within the box. The pattern was identified from the $850 \mathrm{hPa}$ level temperature during the 'Ash Wednesday' fires of $16^{\text {th }}$ February 1983 (Fig. 1a). It was possible to apply the method of Mills (2005) directly to the RCM outputs without any modification, since the RCM output has a temporal and horizontal resolution comparable to the reanalysis products the method is based on. The events with the strongest temperature gradient across the box in the period 1961-1990 were identified from two simulations and the $850 \mathrm{hPa}$ surface of those events is compared to the equivalent chart from the host GCM from Hasson et al. (2008).

Of the two examples of GCM output shown here (Fig. 1b), the event with the strongest temperature gradient identified from one GCM is similar to the Ash Wednesday event, but the other appears structurally different. This indicates that the method detected a pattern that was not reliably associated with prefrontal winds and severe fire weather, and so constitutes a false positive result. This casts doubt on the suitability of using the method directly with GCM output without some modification. The events in the two equivalent RCM examples shown here more closely resemble NCEP reanalysis and are more highly resolved (Fig. 1c). Similar patterns were reproduced in the other four simulations examined (not shown). This indicates that the application of the method may be more appropriate with RCM output, and is worthy of further investigation.

\subsection{FFDI}

The McArthur Mark 5 FFDI was calculated from both daily and 3-hourly outputs from the $\sim 10 \mathrm{~km}$ resolution simulations. The index is given by Equation 1 from Noble et al. (1980):

$$
\mathrm{FFDI}=2 \exp (0.987 \log \mathrm{DF}-0.45+0.0338 \mathrm{~T}+0.0234 \mathrm{~V}-0.0345 \mathrm{RH})),
$$

where DF is a drought factor (1-10), $\mathrm{T}$ is screen temperature $\left({ }^{\circ} \mathrm{C}\right), \mathrm{V}$ is 10 -metre wind speed $\left(\mathrm{km} \mathrm{h}^{-1}\right)$ and $\mathrm{RH}$ is relative humidity (\%). 
Using the 3-hourly output, FFDI time series were calculated in four grid cells approximating the locations of Hobart, Launceston Airport, East Coast north of Coles Bay and West Coast north of Strahan. For this simple illustrative example of sub-daily series, the drought factor was kept at a constant value of the maximum 10. Peak values of FFDI were highest at Hobart, followed by the East Coast, and considerably lower in Launceston and the West Coast, consistent with patterns of high FFDI in observations. Example series of FFDI calculated in this manner for the grid cell of Hobart are shown in Fig 2. The full 30-year series of 3hourly FFDI for 1961-1990 (Fig 2a) shows inter-annual variability in peak values of FFDI, and the example year 1961 (Fig 2b) shows the clear seasonal cycle of FFDI. The mean and variability of FFDI can't be directly compared with observations since the drought factor is kept at 10, however the magnitude of annual peak events (generally FFDI $=30-45$, with some years experiencing events of FFDI $=50-70$ ) compare well with those calculated from Hobart observations by Fox-Hughes (2008).

Three examples of where FFDI exceeded 50 (Fig 2c-e) show the peak events exceed an FFDI of 50 for only one or two 3-hourly periods, which is consistent with observed events of extreme FFDI (Fox-Hughes, In Press). The events are always during warm days, but are more specifically associated with winds speeds in the top percentile and a drop to the very lowest recorded values of relative humidity $(\mathrm{RH}<20 \%$, which is the lowest 0.35 percentile in the model). This supports conventional wisdom that high temperature is a prerequisite for severe fire weather, but high wind and low relative humidity are major drivers of the events of the very worst conditions.

\section{a. $850 \mathrm{hPa}$ temperature $16^{\text {th }}$ February 1983 (NCEP Reanalysis)}

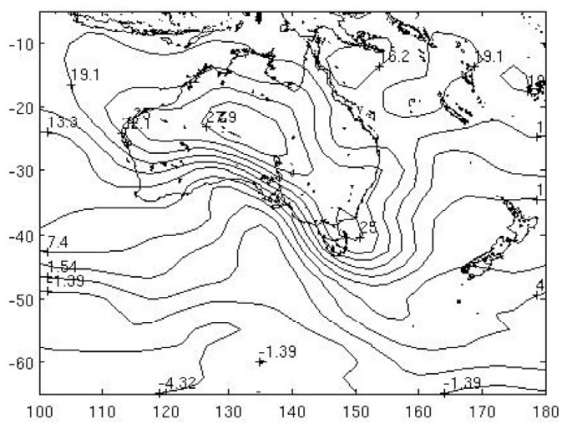

\section{b. GCM Output}
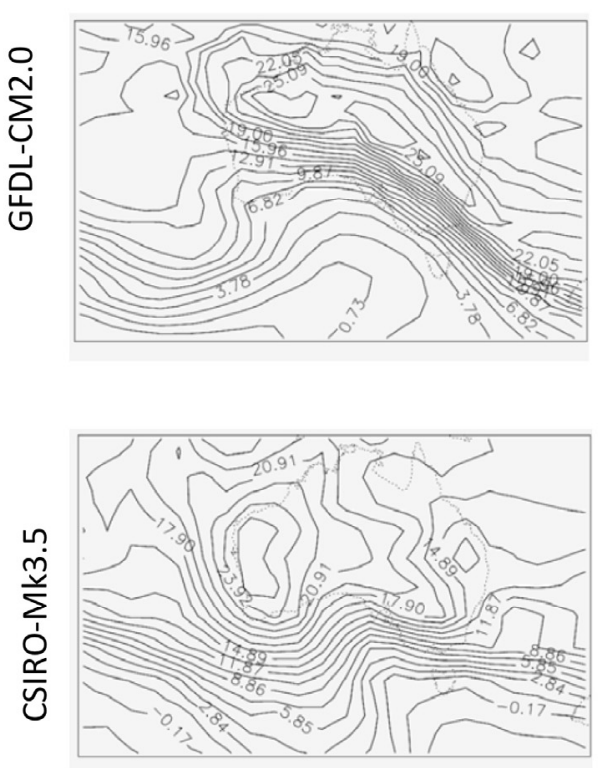

c. RCM Output
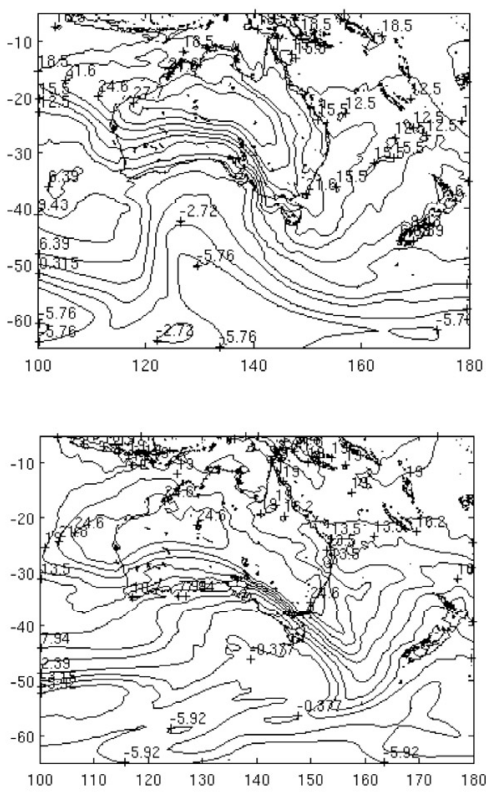

Figure 1. Temperature at the $850 \mathrm{hPa}$ level over the Australian region during synoptic events associated with extreme fire weather detected using the method of Mills (2005), a) NCEP Reanalysis 1 during Ash Wednesday, $16^{\text {th }}$ February 1983 , b) events detected in the 1961-1990 period in GCM output as marked, and c) events detected in the 1961-1990 period in CCAM RCM simulations using the equivalent GCM as input 

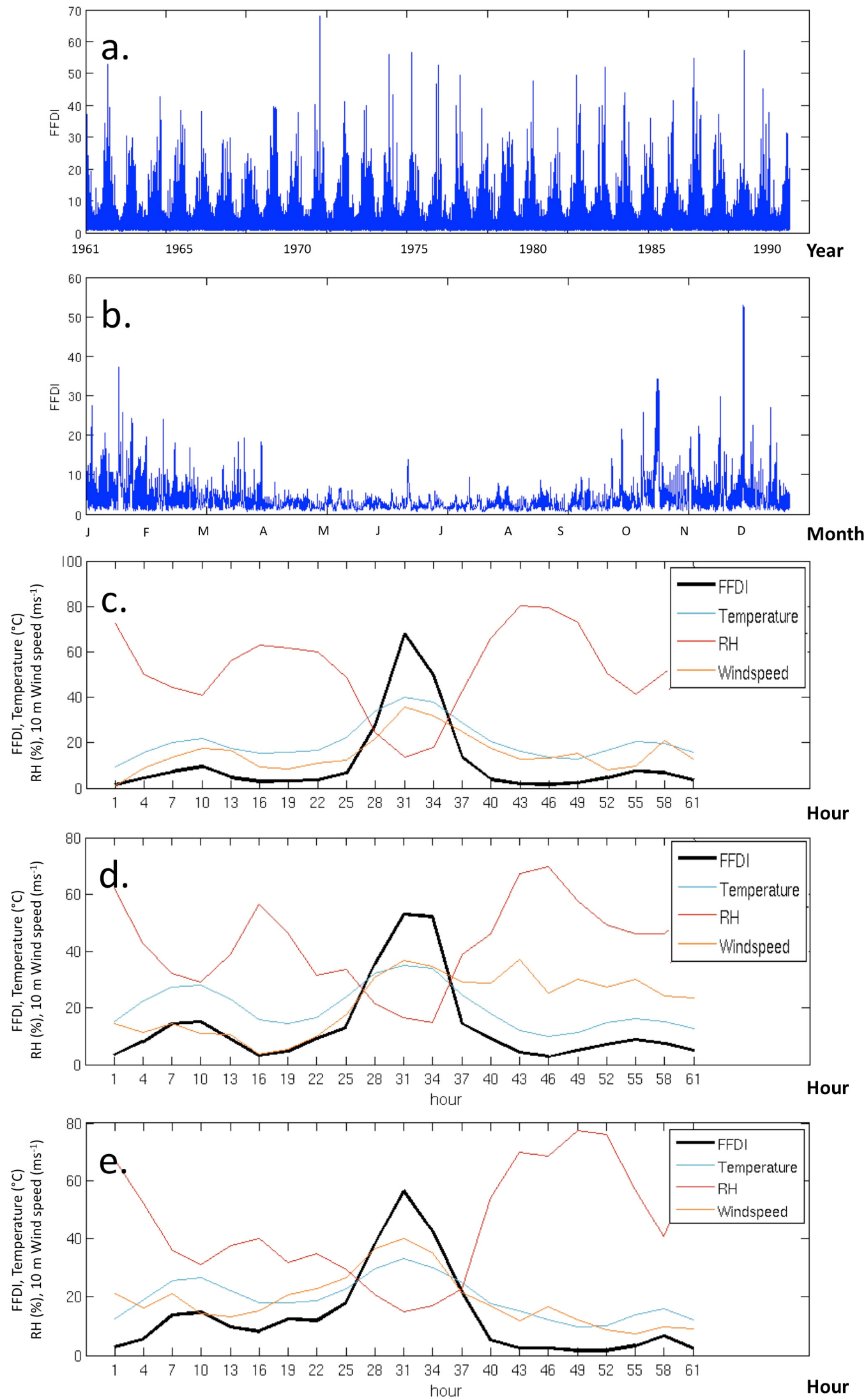

Figure 2. Series of 3-hourly McArthur 5 Forest Fire Danger Index (FFDI) for Hobart in an example model simulation in the baseline climate (CCAM simulation with CSIRO-Mk3.5 as input, 1961-1990) assuming a drought factor of 10 (maximum), a) 3-hourly FFDI for Hobart for the entire period 1961-1990, b) 3-hourly FFDI for an example year 1961, c-e) 3-hourly FFDI series' for the three events of the most extreme fire danger simulated (FFDI $>50$ ), on an arbitrary hourly scale with the peak at hour 31 


\section{RCM CLIMATOLOGIES}

Model output of daily maximum temperature, daily minimum RH and daily maximum 10-metre wind speed are used to generate a climatology of average annual accumulated daily fire danger ( $\Sigma F F D I)$, average annual count of days of FFDI $\geq 25$ (classified as 'Very High' or greater), and the maximum daily FFDI value in any simulation. Values for 1961-1990 are shown in Figure 3, and below each plot are the model mean and standard deviation between models at four locations (Hobart, Launceston Airport, East Coast north of Coles Bay and West Coast north of Strahan, as marked). For the purposes of this preliminary investigation these calculations all use a default drought factor of the maximum 10, and this means that the values of FFDI are artificially high compared to what they would be expected to be using a calculated drought factor. For example, the accumulated FFDI at Hobart of $2764 \pm 38$ and the count of days of FFDI $\geq 25$ of $9 \pm 1$ days year $^{-1}$ are both higher than observations: 1723 and 3.4 days year ${ }^{-1}$ respectively (Hennessy et al. 2005). Incorporating a variable drought factor will result in lower FFDIs that may be more in line with Hennessy et al. (2005). But for the purposes of this paper, the patterns and relative magnitude of these values is of interest here rather than the absolute values.

Values of all three calculations in Figure 3 are highest in the southeast, followed by the east coast, then the north and west. This agrees very well with patterns of measured FFDI (Fox-Hughes 2008). The only location that has a measured FFDI over 100 is the southeast (Fox-Hughes 2008), which is also shown in the model simulation. The maximum FFDI value of 102 in Hobart was recorded in a different model simulation than that shown in Figure 2, hence the difference in peak value seen.

a. $\Sigma$ FFDI

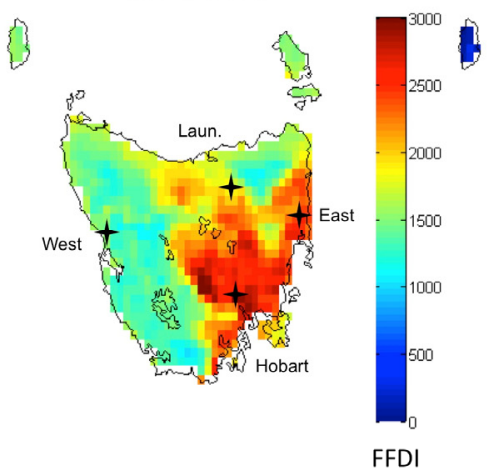

b. Days $\geq$ FFDI 25

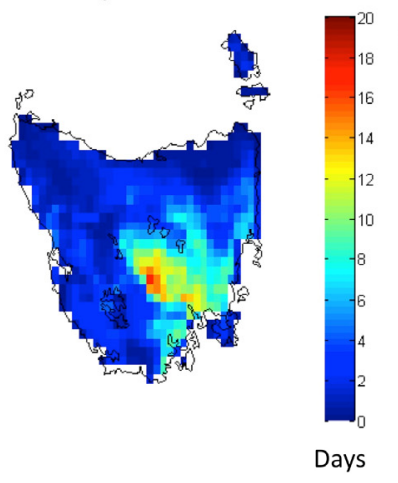

c. Maximum FFDI

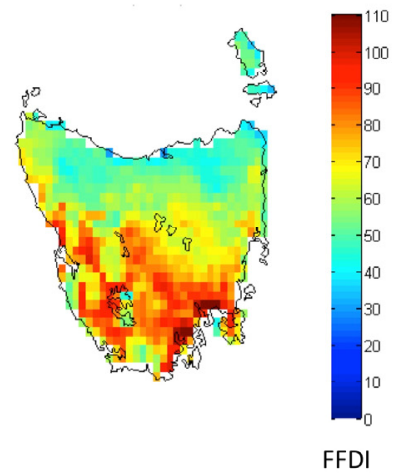

$\begin{array}{ll}\text { Hobart } & 2764 \pm 38 \\ \text { East } & 2608 \pm 35 \\ \text { Laun. } & 1895 \pm 40 \\ \text { West } & 1307 \pm 16\end{array}$

$9 \pm 1$

$7 \pm 0.5$

$3 \pm 0.3$

$2 \pm 0.3$
102

73

58

68

Figure 3. Model mean RCM model outputs of FFDI in Tasmania during 1961-1990, assuming drought factor =10, a) annual accumulated FFDI, b) the number of days of FFDI $\geq 25$ annually and c) the maximum daily FFDI in any of the six simulations. Below each plot are the model mean and model standard deviation between models for at four locations marked with stars (Hobart, East Coast north of Coles Bay, Launceston Airport and West Coast north of Strahan)

\section{DISCUSSION AND CONCLUSIONS}

This preliminary investigation showed that a set of RCM simulations offer several advantages over GCM scale simulations in the modelling of various aspects of bushfire weather. In this way, these results indicate that the output of an RCM is suitable to use for analysis of bushfire dynamics and incidents of elevated fire danger indices with a change in climate.

The RCM were shown to produce more realistic simulations of one atmospheric pattern associated with elevated fire danger in Tasmania compared to GCM outputs. The realistic simulation of this one diagnostic of one pattern (from Mills 2005) is taken as evidence that the RCM shows promise as a tool for investigating changes to bushfire weather dynamics with climate change, so further investigation will be undertaken. 
Grose et al., Bushfire conditions under a warming climate - the value of regional climate modelling

The RCM also allows analysis at sub-daily time scales (3 hourly) and output variables that are directly equivalent to observations (e.g. daily minimum RH not mean) to calculate fire indices. The RCM was able to faithfully reproduce some important spatial and temporal patterns in FFDI over Tasmania. The temporal pattern FFDI at time scales from sub-daily to annual in Hobart appeared plausible compared to observations. This is in contrast to GCM outputs, which do not currently include sub-daily outputs and the quality of the coarse-scale model output was not considered sufficient for the analysis of FFDI directly by Hennessey et al. (2005). The spatial patterns of daily accumulated FFDI, count of days FFDI $>25$ and maximum FFDI compared well to observations. This is also in contrast to GCM outputs, which can't indicate regional detail of fire danger (featuring only 0-6 grid cells over Tasmania). The typical values of FFDI generated by the RCM were also plausible considering the simplified method used (assuming drought factor of 10).

The results of this analysis are presented as a preliminary assessment of using direct RCM outputs to examine trends in fire risk, and to justify further investigation. The results suggest that analysis of fire risk may be possible using an event-based approach, rather than merely examining a change in averages or percentiles in fire indices or their input variables or using modified observed datasets. For the calculation of fire indices, any correction factors (or bias correction) of input variables would be relatively small. RCM outputs appear to show some advantages over the use of raw GCM outputs, and with further careful analysis, these RCMs may be a useful tool for examining questions of future fire risk with a changing climate. The next obvious step is to start incorporating a real drought factor based on rainfall.

\section{ACKNOWLEDGMENTS}

The authors would like to acknowledge the contribution from the Climate Futures for Tasmania project for the provision of the model simulations, particularly Stuart Corney, Jack Katzfey and John McGregor. This work was supported by the Australian Government's Cooperative Research Centre Program through the Antarctic Climate and Ecosystems Cooperative Research Centre (ACE CRC). Climate Futures for Tasmania was possible with support through funding and research from a consortium of state and national partners.

\section{REFERENCES}

Bradstock, R.A., J.S. Cohn, A.M. Gill, M. Bedward and C. Lucas (2009). "Prediction of the probability of large fires in the Sydney region of south-eastern Australia using fire weather." International Journal of Wildland Fire 18: 932-943.

Corney, S.P., J.K. Katzfey, J.L. McGregor, M.R. Grose, J. Bennett, C.J. White, G.K. Holz, S. Gaynor and N.L. Bindoff (2010). Climate Futures for Tasmania: climate modelling. Hobart, Tasmania, Antarctic Climate and Ecosystems Cooperative Research Centre.

Crimmins, M.A., and A.C. Comrie (2004). Interactions between antecedent climate and wildfire variability across south-east Arizona. International Journal of Wildland Fire. 13, 455-466

Fox-Hughes, P. (2008). A fire danger climatology for Tasmania. Australian Meteorological Magazine. 57: 109-120

Fox-Hughes, P. (In Press). Springtime fire weather in Tasmania, Australia: two case studies. Journal of Applied Mathematics and Computing Accepted subject to review 22/4/2011

Hasson, A.E.A., G.A. Mills, B. Timbal and K.J.E. Walsh (2008). Assessing the impact of climate change on extreme fire weather in southeast Australia. CAWCR Technical Report. Melbourne, Australia, The Centre for Australian Weather and Climate Research. 007: 86.

Hennessy, K.J., C. Lucas, N. Nicholls, J. Bathols, R. Suppiah and J. Ricketts (2005). Climate change impacts on fire-weather in south-east Australia. CSIRO Marine and Atmospheric Research, Bushfire CRC and Bureau of Meteorology: 91.

King, K.J., R.M. de Ligt and G.J. Cary (2009). Changes in fire and carbon dynamics for projected future climates in the southeastern Australian high country. International Congress on Modelling and Simulation (MODSIM 2009 ), ed. Anderssen, R.S., R.D. Braddock and L.T.H. Newham, The Modelling and Simulation Society of Australia and New Zealand Inc. and the International Association for Mathematics and Computers in Simulation, Australia, pp. 25692575

McArthur, A.G. (1967). Fire behaviour in eucalypt forest. Commonwealth of Australia Timber Bureau Leaflet 107: 25

Noble I.R., G.A.A Bary and A.M Gill (1980). McArthur's fire danger meters expressed as equations. Australian Journal of Ecology, 5: 201-203.

Mills, G.A. (2005). A re-examination of the synoptic and mesoscale meteorology of Ash Wednesday 1983. Australian Meteorological Magazine 54: 35-55.

Williams, A.A.J., D.J. Karoly and N Tapper (2001). The sensitivity of Australian fire danger to climate change. Climatic Change 49(1-2): 171-191. 